\title{
The Effect and Mechanism of
} Cholesterol and Vitamin $B_{12}$ on Multi-Domain Cognitive Function: A Prospective Study on Chinese Middle-Aged and Older Adults

\author{
Lijing Wang ${ }^{\dagger}$, Kuo Liu ${ }^{\dagger}$, Xiaona Zhang, Yushan Wang, Wen Liu, Tao Wang, Ling Hao, \\ Mengwei Ju and Rong Xiao*
}

School of Public Health, Capital Medical University, Beijing, China

Background: Nutrients are associated with cognitive function, but limited research studies have systematically evaluated on multi-domain cognitive function. The aim of this study was to investigate the effect and mechanism of specific nutrient on multi-domain cognitive function, and provide nutrition guidance for improving cognitive function.

OPEN ACCESS

Edited by:

Stephanie R. Rainey-Smith, Murdoch University, Australia

Reviewed by: Mitsuru Shinohara, National Center for Geriatrics and Gerontology (NCGG), Japan

Beita Zhao,

Northwest $A$ and F University, China

*Correspondence:

Rong Xiao

xiaor22@ccmu.edu.cn

these authors have contributed equally to this work and share first authorship

Received: 11 May 2021 Accepted: 20 July 2021 Published: 27 August 2021

Citation:

Wang L, Liu K, Zhang X, Wang Y,

Liu W, Wang T, Hao L, Ju M and Xiao R (2021) The Effect and Mechanism of Cholesterol and Vitamin

$B_{12}$ on Multi-Domain Cognitive Function: A Prospective Study on Chinese Middle-Aged and Older

Adults.

Front. Aging Neurosci. 13:707958.

doi: 10.3389/fnagi.2021.707958
Methods: Participants were selected based on a multicenter prospective study on middle-aged and older adults in China. Global cognitive function was evaluated by the Mini-Mental State Examination (MMSE). Nutrients intake was assessed according to food frequency questionnaire and China Food Composition Database, and principal component analysis was performed to extract nutrient patterns. Associations between specific nutrients and cognitive function were assessed using log-binomial regression. Restricted cubic spline was used to illustrate the dose-response relationship of nutrients with multi-domain cognitive function. Mediation analysis was used to determine the mechanism of nutrients in cognitive function.

Results: Four nutrient patterns were identified (vitamin-mineral, protein-carbohydrate, fatty acid-vitamin $\mathrm{E}$, and cholesterol-vitamin $\mathrm{B}_{12}$ ), and only a nutrient pattern rich in cholesterol and vitamin $B_{12}$ was found associated with cognitive function $(R R=0.891$, $95 \% \mathrm{Cl}=0.794-0.999)$. In multi-domain cognitive function, dietary cholesterol and vitamin $\mathrm{B}_{12}$ were related to better performance of visual memory function $(P=0.034$, $P=0.02)$. In dose-response relationship, it suggested a $U$-shaped association between vitamin $\mathrm{B}_{12}$ and MMSE $(P=0.02)$ within a certain range.

Conclusions: Dietary intake rich in cholesterol and vitamin $\mathrm{B}_{12}$ was associated with better cognitive function, and vitamin $\mathrm{B}_{12}$ had a $U$-shaped dose-response relation with MMSE. Thus, ensuring moderate cholesterol and vitamin $\mathrm{B}_{12}$ intake may be an advisable strategy to improve cognitive function in middle-aged and older adults.

Clinical Trial Registration: EMCOA, ChiCTR-OOC-17011882, Registered 5th, July 2017-Retrospectively registered, http://www.medresman.org/uc/project/projectedit. aspx?proj=2610

Keywords: mild cognitive impairment, cholesterol, vitamin B12, dose-response relation, mediation analysis 


\section{INTRODUCTION}

Alzheimer's disease is one of the most common neurodegenerative diseases, characterized by progressive impairment in cognitive function, learning ability, memory function, and executive reasoning (Cui et al., 2020). Mild cognitive impairment (MCI) is a transitional stage between normal aging and dementia, 60-100\% can develop into Alzheimer's disease (AD) within 5-10 years (Xue et al., 2018). According to the latest research, the overall prevalence of MCI and $\mathrm{AD}$ was estimated to be 15.5 and $3.9 \%$, respectively, which imposed a considerable burden on individuals and society in China (Jia et al., 2020). Thus, early intervention on cognitive function can slow down the progress of $\mathrm{AD}$ efficiently (Fiorini et al., 2020).

In the past two or three decades, dietary habits in China have been experiencing a great change, particularly consumption of meat and sodium (Zhou et al., 2019). For example, residents in Beijing experienced a transition from traditional grain-based to animal-sourced diet (Xiong et al., 2019). Meantime, dietary habit transition leads to change in nutrient intake. Various studies have verified a strong link between nutrients and cognitive function, and most studies only focused on a single nutrient, while a single nutrient was not sufficient to affect cognitive function (Shi et al., 2019). Nutrient pattern may be more appropriate to examine the cumulative beneficial effect and common mechanism of nutrients in cognitive function, and it could explore the bioactive components of foods and potential interactions (Scarmeas et al., 2018). In Sweden, recent studies have found that nutrient pattern may play important roles in brain heath, but it still lacked relevant research in Chinese populations (Prinelli et al., 2019). Besides, the effects of nutrients on cognitive function may vary with intake doses, which revealed potential non-linear relationships between nutrients and cognitive function (Chianese et al., 2017). A dose-response meta-analysis showed a significant non-linear relationship between polyunsaturated fatty acids and MCI (Zhang et al., 2016). A prospective cohort study also found a non-linear relationship between dietary $\mathrm{Mg}$ intake and cognitive function in adult women aged 65-79 in the United States (Lo et al., 2019). Therefore, it is necessary to focus on the non-linear relationship between nutrients and cognitive function to provide a precise dietary recommendation.

\footnotetext{
Abbreviations: 27-OHC, 27-hydroxycholesterol; 24S-OHC, 24Shydroxycholesterol; ACME, average causal mediation effect; AD, Alzheimer's disease; $A \beta, \beta$-amyloid; AVLT-LR, auditory verbal learning test-long recall; AVLTIR, auditory verbal learning test-immediate recall; AVLT-SR, auditory verbal learning test-short recall; BMI, body mass index; CFCD, China Food Composition Database; CVD, cerebrovascular diseases; DSTB, Digit span backwards; DSTF, Digit span forwards; EMCOA, effects and mechanism of cholesterol and oxysterol on Alzheimer's disease; FFQ, Food Frequency Questionnaire; LMT-IR, logical memory test-immediate recall; MCI, mild cognitive impairment; MMSE, MiniMental State Examination; MUFA, monounsaturated fatty acid; NC, normal cognition; PCA, principal component analysis; PMT, picture memories test; $\mathrm{p}$-tau, phosphorylated tau; PUFA, polyunsaturated fatty acid; RCA, restricted cubic spline; SCWT-IT, Stroop Color-Word Test Interference Trial; SFA, saturated fatty acid; TMT, Trail Making Test; $\mathrm{t}$-tau, total tau; WMS-RC, Wechsler Memory Scale-Revised of China.
}

Nowadays, increasing studies elucidated the mechanisms of nutrients affecting cognitive function, such as oxidative stress, inflammatory processes, and $\mathrm{A} \beta$ or tau protein accumulation (Poulose et al., 2017). Recently, cholesterol and its oxygenated derivatives were found to play an important role in cognitive impairment, such as 27-hydroxycholesterol (27-OHC) and 24S-hydroxycholesterol (24S-OHC) (Wang et al., 2016a). An animal research study proved that high fat/cholesterol diet resulted in increasing level of tau phosphorylation and memory loss (Bhat and Thirumangalakudi, 2013). Because of their role in homocysteine metabolism, the mechanisms of vitamin $\mathrm{B}_{12}$ affecting cognitive function were briefly summarized as cerebrovascular effects, activation of tau kinases, or inhibition of methylation reactions (Smith and Refsum, 2016). It showed that both cholesterol and vitamin $\mathrm{B}_{12}$ could affect cognitive function through tau phosphorylation; however, the findings are inconsistent and fragmented (Vauzour et al., 2017). Therefore, it is necessary to perform mediation analysis to verify the role of cholesterol oxygenated derivatives or tau phosphorylation between nutrients and cognitive function, and to provide an important basis for exploring the common mechanism between nutrients and cognitive function.

This study was performed mainly to demonstrate the dose-response relationship between specific nutrient in nutrient patterns and multi-domain cognitive function based on a multicenter prospective study, and explore the potential mechanisms, which could provide scientific dietary recommendations for middle-aged and older Chinese populations.

\section{METHODS}

\section{Participants}

The Effects and Mechanism Investigation of Cholesterol and Oxysterol on Alzheimer's disease (EMCOA) study was a multicenter prospective study on community-dwelling volunteers initiated by Capital Medical University in 20142015 (Yu et al., 2018). In this study, participants aged 50 and 70 years were recruited and followed for an average of 2 years. Participants who had neuropsychiatric problems (e.g., depression, schizophrenia, drug addiction or other), severe diseases (e.g., visual impairment, hearing loss, or other severe organ dysfunction), who used dietary supplements or drugs affecting cognitive function, who were untraceable or failure to accomplish were excluded. Finally, 2,546 middle-aged and older eligible adults were selected for the demonstration of the relationship between specific nutrients in nutrient pattern, food composition, and multi-domain cognitive function. A subgroup that included 104 participants was randomly selected to explore the mechanism of nutrients in cognitive function. The details are shown in Figure 1. Face-to-face interviews were conducted by a survey team composed of clinical neuropsychologists and research investigators who have been trained in the details of the measurements and questionnaires. The assessments of diet and cognitive function was conducted initially, and then after 2 years. The EpiData software was used for parallel double data entry. 


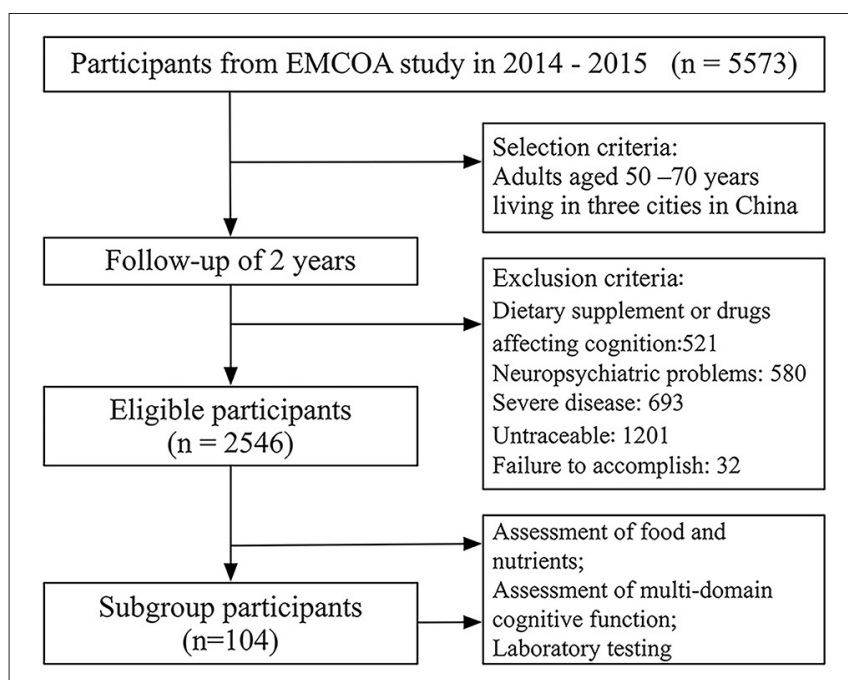

FIGURE 1 | Flow diagram. by neurologists to establish a clinical diagnosis (Wong et al., 2016; Yin et al., 2018; Jiang et al., 2020).

Comprehensive neuropsychological measures were used to assess the multi-domain cognitive function ( $\mathrm{Yu}$ et al., 2018). Verbal memory function was assessed by Auditory Verbal Learning Test (AVLT) using summarized scores of immediate recall (AVLT-IR), short recall (AVLT-SR), and long recall (AVLT-LR) (Hong et al., 2015); attention/processing speed/executive function was assessed by Logical Memory Test-immediate recall (LMT-IR) (Wang et al., 2016b), Digit Span Forward (DSTF), and Digit Span Backward (DSTB) (Darby et al., 2017), and Trail Making Tests (TMT) A and B (Wei et al., 2018) from Wechsler Memory Scale Revised for China (WMS-RC); flexibility of cognitive function was assessed by the Stroop Color-Word Test-Interference Trial (SCWT-IT) (Bondi et al., 2002); and visual memory function was assessed by the Picture Memories Test (PMT) from WMS-RC (Krakowski and Czobor, 2011). The measurements were performed in a quiet and private room by trained technicians.

\section{Assessment of Food Composition and Nutrients}

Dietary intake was assessed with a semiquantitative Food Frequency Questionnaire (FFQ) (Clare et al., 2017), which included 85 different foods types of 33 items. The participants were requested to state the frequency (per year, month, week, or day) and amount (in grams, bowls, etc.) of food intake for each food item on baseline, and follow up to make sure they were on the same food regimen during the study. For each food reported, food models and an album with over 50 photos of the most common dietary products were used as helpful tools to determine the amounts of food portions. Nutrient intake was calculated by multiplying the nutrient content of each food of specific portion size by the frequency of consumption and then summed over all food items from the China Food Composition Database (CFCD) (Rong et al., 2017). In this study, the following nutrient intake was calculated: protein, carbohydrate, dietary fiber, cholesterol, saturated fatty acid (SFA), monounsaturated fatty acid (MUFA), polyunsaturated fatty acid (PUFA), vitamin $A$, vitamin $B_{1}$, vitamin $B_{2}$, niacin, vitamin $B_{6}$, folic acid, vitamin $B_{12}$, vitamin $C$, vitamin $E$, magnesium, iron, zinc, selenium, copper, and manganese. The food composition included meat, milk and its products, eggs, grain, fruits, vegetables, etc.

\section{Assessment of Cognitive Function}

The global cognitive function of the participants was assessed by Mini-Mental State Examination (MMSE). MMSE is commonly used as a simple tool to screen for cognitive impairment based on education level. The diagnostic criteria used for MCI screening were as follows: (1) illiterate individuals $\leq 19$ points, (2) individuals with 6 or less years of education $\leq 22$ points, (3) individuals with 7 or more years of education $\leq 26$ points. Then, if the participants were suspected of having MCI based on their MMSE performance, they would be comprehensively examined

\section{Covariates}

The questionnaires on sociodemographic included age, sex, education (in years), body mass index (BMI); lifestyle risk factors included smoking, drinking, and reading habit; and medical history included hypertension (measured blood pressure > 140/90 $\mathrm{mmHg}$ or antihypertensive medication), hyperlipidemia (total cholesterol $\geq 6.2 \mathrm{mmol} / \mathrm{L}$ or low-density lipoprotein $\geq 4.1 \mathrm{mmol} / \mathrm{L}$ or high-density lipoprotein $<1 \mathrm{mmol} / \mathrm{L}$ or triglycerides $\geq 2.3 \mathrm{mmol} / \mathrm{L}$ or lipid-lowering medication), diabetes (fasting glucose $\geq 7 \mathrm{mmol} / \mathrm{L}$ or antidiabetic medication), and cerebrovascular diseases (CVD). Smokers were defined as those who have smoked at least one cigarette per day in the last 6 months. Alcohol drinkers were defined as those who have consumed any type of alcoholic beverage at least once a week in the last 6 months. Weight and height were measured according to World Health Organization recommendations, and BMI was calculated as weight in kilograms divided by height in meters squared $\left(\mathrm{kg} / \mathrm{m}^{2}\right)$ (Physical Status, 1995).

\section{Laboratory Testing}

After at least $12 \mathrm{~h}$ of fasting, blood samples were collected in $6 \mathrm{ml}$ tubes containing coagulant and inverted 8-10 times. Serum was obtained by centrifugation at $3,000 \mathrm{rpm}$ at $4^{\circ} \mathrm{C}$ for $10 \mathrm{~min}$, and stored at $-80^{\circ} \mathrm{C}$ until use. The serum concentrations of 27 $\mathrm{OHC}$ and $24 \mathrm{~S}-\mathrm{OHC}$ were measured by high performance liquid chromatography-mass spectrometry (HPLC-MS). A standard curve was generated and used to calculate the concentrations of 27-OHC and 24S-OHC with internal standard, according to the method previously described (Bandaru and Haughey, 2014). Serum total tau (t-tau) and phosphorylated tau ( $\mathrm{p}$-tau) were measured using a commercial enzyme-linked immunosorbent assay (ELISA) kit, and the concentration of the samples was determined by comparing the standard curve (Tang et al., 2019). 


\section{Statistical Analysis}

Statistical analyses were performed using STATA version 15.0 (Stata Corp, College Station, TX, United States), R statistical software version 3.6.1 (R Foundation, Vienna, Austria), and IBM SPSS Statistics 23.0 software (SPSS, Chicago, IL, United States). A two-sided $P \leq 0.05$ was considered statistically significant.

Continuous variables were expressed as median (interquartile range) and compared by Mann-Whitney $U$-test. Categorical variables were presented as number (percentages) and compared by chi-square test or Fisher's exact test. Principal component analysis (PCA) was performed to identify nutrient patterns. Varimax rotation was used in component analysis, and eigenvalues $>1$ were considered as principal component (Makura-Kankwende et al., 2020). Log-binomial regression was used to estimate the relationship between nutrient patterns and MCI (Palta et al., 2018). Multiple linear regression was used to illustrate the linear relationship between specific nutrients in nutrient patterns and multi-domain cognitive function. Restricted cubic spline (RCA) was used to flexibly model the dose-response relationship and explore the potential non-linear association between specific nutrients in nutrient patterns and multi-domain cognitive function (Desquilbet and Mariotti, 2010).

\section{RESULTS}

\section{Characteristics of Participants}

General characteristics, nutrients intake, and multidomain cognitive function were compared between participants with mild cognitive impairment (MCI) and those with normal cognition (NC). The details are shown in Supplementary Table 1 . Of the 2,546 participants, $54.6 \%$ were women. It showed that the difference of age between the MCI and NC groups was significant, but the median was same $(P$ $=0.016$ ). Compared with NC, participants in the MCI group had a worse performance in multi-domain cognitive function $(P<0.001)$. No significant differences were observed in the other characteristics.

\section{Association Between Nutrient Patterns and $\mathrm{MCl}$}

Four independent nutrient patterns were identified by principal component analysis (PCA) (Table 1). The first nutrient pattern was identified as "Vitamin-Mineral," explained $36.4 \%$ of the variance and was illustrated by intakes of vitamin $A$, vitamin $B_{2}$, vitamin $B_{6}$, folic acid, vitamin $C$, magnesium, iron, zinc, copper, and manganese. The second nutrient pattern was identified as "Protein-Carbohydrate," explained $21.6 \%$ of the variance, and was illustrated by intakes of protein, carbohydrate, dietary fiber, and vitamin $B_{1}$. The third nutrient pattern was identified as "Fatty Acid-Vitamin E," explained $17.2 \%$ of the variance, and was illustrated by intakes of SFA, MUFA, PUFA, and vitamin E. The fourth nutrient pattern was identified as "CholesterolVitamin $B_{12}$," explained $12.2 \%$ of the variance, and was illustrated by intakes of cholesterol and vitamin $B_{12}$.

Log-binomial regression was used to determine the effects of the different nutrient patterns on mild cognitive impairment
TABLE 1 | Principal component analysis of nutrient patterns.

\begin{tabular}{lcccc}
\hline Variables & $\begin{array}{c}\text { Vitamin- } \\
\text { mineral }\end{array}$ & $\begin{array}{c}\text { Protein- } \\
\text { carbohydrate }\end{array}$ & $\begin{array}{c}\text { Fatty acid- } \\
\text { vitamin } \mathbf{E}\end{array}$ & $\begin{array}{c}\text { Cholesterol- } \\
\text { vitamin } \mathbf{B}_{\mathbf{1 2}}\end{array}$ \\
\hline Protein & 0.260 & $\mathbf{0 . 7 0 2}$ & 0.401 & 0.449 \\
Carbohydrate & 0.145 & $\mathbf{0 . 9 4 5}$ & 0.079 & 0.083 \\
Dietary fiber & 0.261 & $\mathbf{0 . 8 4 1}$ & 0.112 & -0.027 \\
Cholesterol & 0.036 & 0.159 & 0.132 & $\mathbf{0 . 8 4 2}$ \\
SFA & 0.040 & 0.191 & $\mathbf{0 . 7 3 5}$ & 0.574 \\
MUFA & 0.051 & 0.068 & $\mathbf{0 . 9 6 5}$ & 0.016 \\
PUFA & 0.021 & 0.204 & $\mathbf{0 . 8 1 6}$ & 0.429 \\
Vitamin A & $\mathbf{0 . 9 0 0}$ & 0.146 & -0.024 & 0.225 \\
Vitamin $\mathrm{B}_{1}$ & 0.392 & $\mathbf{0 . 8 1 0}$ & 0.169 & 0.253 \\
Vitamin $\mathrm{B}_{2}$ & $\mathbf{0 . 8 6 4}$ & 0.260 & 0.085 & 0.275 \\
Niacin & 0.600 & 0.442 & 0.480 & 0.206 \\
Vitamin $\mathrm{B}_{6}$ & $\mathbf{0 . 9 2 2}$ & 0.210 & 0.072 & 0.063 \\
Folic acid & $\mathbf{0 . 9 1 7}$ & 0.317 & 0.151 & 0.068 \\
Vitamin $\mathrm{B}_{12}$ & 0.121 & 0.013 & 0.066 & $\mathbf{0 . 8 6 1}$ \\
Vitamin C & $\mathbf{0 . 9 4 4}$ & 0.184 & -0.017 & -0.022 \\
Vitamin E & 0.259 & 0.185 & $\mathbf{0 . 8 7 2}$ & -0.058 \\
Magnesium & $\mathbf{0 . 6 7 1}$ & 0.524 & 0.451 & 0.093 \\
Iron & $\mathbf{0 . 9 0 3}$ & 0.385 & 0.084 & 0.055 \\
Zinc & $\mathbf{0 . 7 0 6}$ & 0.556 & 0.283 & 0.284 \\
Selenium & 0.579 & 0.507 & 0.251 & 0.521 \\
Copper & $\mathbf{0 . 7 6 3}$ & 0.519 & 0.322 & 0.096 \\
Manganese & $\mathbf{0 . 6 9 3}$ & $\mathbf{0 . 6 1 1}$ & 0.304 & 0.033 \\
lodine & 0.552 & -0.055 & 0.070 & -0.062 \\
\hline Bolden & & &
\end{tabular}

Bold entries indicate measures with high loadings on each factor.

SFA, saturated fatty acid; MUFA, monounsaturated fatty acid; PUFA, polyunsaturated fatty acid.

(MCI). The results showed that only the Cholesterol-Vitamin $\mathrm{B}_{12}$ nutrient pattern $(\mathrm{RR}=0.891,95 \% \mathrm{CI}=0.794-.999, P=0.048)$ was negatively associated with MCI (Table 2).

\section{Association Between Cholesterol or Vitamin $B_{12}$ and Multi-Domain Cognitive Function}

Multiple linear regression models were used to further examine the association between baseline cholesterol or vitamin $B_{12}$ and follow-up multi-domain cognitive function. The models were adjusted for age, sex, education, BMI, smoking, drinking, hypertension, hyperlipidemia, diabetes, CVD, and other nutrient patterns (Table 3). They showed that cholesterol was positively associated with PMT $(\beta=0.137 ; P=0.034)$, and that vitamin $B_{12}$ was also related to better performance in PMT $(\beta=0.08 ; P=0.02)$.

Restricted cubic spline analysis was performed to simulate the non-linear dose-response relationship between cholesterol or vitamin $\mathrm{B}_{12}$ and multi-domain cognitive function. The models were adjusted for age, sex, education, BMI, smoking, drinking, hypertension, hyperlipidemia, diabetes, CVD, and other nutrient patterns (Table 4). The results showed that vitamin $B_{12}$ had a non-linear relationship with MMSE $(P=0.020)$. Regarding the dose-response curve, it emerged a $U$-shaped relationship 
TABLE 2 | Log-binomial regression of nutrient patterns and MCl.

\begin{tabular}{|c|c|c|c|c|}
\hline \multicolumn{2}{|c|}{ Nutrient patterns } & \multicolumn{2}{|c|}{ RR $(95 \% \mathrm{Cl})$} & $P$-value \\
\hline \multicolumn{5}{|c|}{ Unadjusted } \\
\hline \multicolumn{2}{|c|}{ Vitamin-mineral } & \multicolumn{2}{|c|}{$1.024(0.933,1.122)$} & 0.622 \\
\hline \multicolumn{2}{|c|}{ Protein-carbohydrate } & \multicolumn{2}{|c|}{$0.970(0.877,1.073)$} & 0.557 \\
\hline \multicolumn{2}{|c|}{ Fatty acid-vitamin E } & \multicolumn{2}{|c|}{$0.994(0.901,1.097)$} & 0.907 \\
\hline \multicolumn{2}{|c|}{ Cholesterol-vitamin $\mathrm{B}_{12}$} & \multicolumn{2}{|c|}{$0.892(0.796,1.000)$} & 0.051 \\
\hline \multicolumn{5}{|l|}{ Model 1} \\
\hline \multicolumn{2}{|c|}{ Vitamin-mineral } & \multicolumn{2}{|c|}{$1.016(0.926,1.115)$} & 0.734 \\
\hline \multicolumn{2}{|c|}{ Protein-carbohydrate } & \multicolumn{2}{|c|}{$0.967(0.873,1.072)$} & 0.527 \\
\hline \multicolumn{2}{|c|}{ Fatty acid-vitamin E } & \multicolumn{2}{|c|}{$0.993(0.900,1.095)$} & 0.885 \\
\hline \multicolumn{2}{|c|}{ Cholesterol-vitamin $\mathrm{B}_{12}$} & \multicolumn{2}{|c|}{$0.894(0.797,1.003)$} & 0.055 \\
\hline \multicolumn{5}{|l|}{ Model 2} \\
\hline \multicolumn{2}{|c|}{ Vitamin-mineral } & \multicolumn{2}{|c|}{$1.021(0.931,1.119)$} & 0.665 \\
\hline \multicolumn{2}{|c|}{ Protein-carbohydrate } & \multicolumn{2}{|c|}{$0.963(0.868,1.068)$} & 0.477 \\
\hline \multicolumn{2}{|c|}{ Fatty acid-vitamin E } & \multicolumn{2}{|c|}{$0.994(0.902,1.096)$} & 0.906 \\
\hline \multicolumn{2}{|c|}{ Cholesterol-vitamin $\mathrm{B}_{12}$} & \multicolumn{2}{|c|}{$0.891(0.794,0.999)$} & $0.048^{*}$ \\
\hline \multicolumn{5}{|c|}{$\begin{array}{l}\text { Model } 1 \text { was adjusted for age, sex, education, BMI, smoking, and drinking; Model } 2 \text { was } \\
\text { adjusted for Model } 1 \text { and hypertension, hyperlipidemia, diabetes, and CVD. } \\
\text { MCl, mild cognitive impairment; BMI, body mass index; CVD, cerebrovascular diseases; } \\
R R \text {, risk ratio; Cl, confidence interval. } \\
{ }^{*} P<0.05,{ }^{\star *} P<0.001 \text {. }\end{array}$} \\
\hline \multicolumn{5}{|c|}{$\begin{array}{l}\text { TABLE } 3 \text { | Linear relationship between cholesterol or vitamin } B_{12} \text { and } \\
\text { multi-domain cognitive function. }\end{array}$} \\
\hline Variables & Cholesterol & & Vitamin $B_{12}$ & \\
\hline & $\beta(95 \% \mathrm{Cl})$ & $P$-value & $\beta(95 \% \mathrm{Cl})$ & $P$-value \\
\hline MMSE & $0.024(-0.053,0.100)$ & 0.547 & $0.023(-0.018,0.064)$ & 0.269 \\
\hline AVLT-IR & $0.024(-0.153,0.200)$ & 0.792 & $0.027(-0.067,0.122)$ & 0.567 \\
\hline AVLT-SR & $0.072(-0.020,0.164)$ & 0.125 & $0.037(-0.012,0.086)$ & 0.139 \\
\hline AVLT-LR & $0.005(-0.098,0.108)$ & 0.930 & $0.003(-0.058,0.052)$ & 0.915 \\
\hline SDMT & $0.154(-0.253,0.560)$ & 0.459 & $-0.012(-0.229,0.205)$ & 0.913 \\
\hline LMT & $-0.029(-0.234,0.177)$ & 0.784 & $0.034(-0.076,0.143)$ & 0.544 \\
\hline TMTA & $0.018(-0.898,0.934)$ & 0.970 & $0.045(-0.444,0.535)$ & 0.856 \\
\hline TMTB & $-0.640(-3.255,1.975)$ & 0.631 & $-0.326(-1.723,1.071)$ & 0.647 \\
\hline DSTF & $-0.010(-0.061,0.041)$ & 0.697 & $0.013(-0.014,0.040)$ & 0.353 \\
\hline DSTB & $-0.027(-0.074,0.020)$ & 0.260 & $-0.019(-0.044,0.007)$ & 0.149 \\
\hline PMT & $0.137(0.010,0.263)$ & $0.034^{\star}$ & $0.080(0.013,0.148)$ & $0.020^{\star}$ \\
\hline SCWT-IT & $0.447(-0.284,1.179)$ & 0.230 & $0.042(-0.348,0.432)$ & 0.832 \\
\hline
\end{tabular}

Adjusted for age, sex, education, BMI, smoking, drinking, hypertension, hyperlipidemia, diabetes, CVD, and other nutrient patterns using multiple linear regression models. MMSE, Mini-Mental State Examination; AVLT-IR, Auditory Verbal Learning Testimmediate recall; AVLT-SR, Auditory Verbal Learning Test-short recall; AVLT-LR Auditory Verbal Learning Test-long recall; SDMT, Symbol Digit Modalities Test; LMT, Logical Memory Test; TMTA (B), Trail Making Test A (B); DSTF, digit span test forwards; DSTB, digit span test backwards; PMT, Picture Memories Test; SCWT-IT, Stroop Color-Word Test Interference Trial; BMI, body mass index; CVD, cerebrovascular diseases; Cl, confidence interval.

${ }^{\star} P<0.05 .{ }^{\star \star} P<0.001$.

between vitamin $B_{12}$ and MMSE when the intake was $<3 \mu \mathrm{g} /$ day (Figure 2). No dose-response relationship between cholesterol and multi-domain cognitive function was found.
TABLE 4 | Dose-response relationship between cholesterol or vitamin $B_{12}$ and multi-domain cognitive function.

\begin{tabular}{lccccc}
\hline \multirow{2}{*}{ Variables } & \multicolumn{2}{c}{ Cholesterol } & & \multicolumn{2}{c}{ Vitamin $\mathbf{B}_{\mathbf{1 2}}$} \\
\cline { 2 - 3 } \cline { 5 - 6 } & P-overall & P-non-linear & & P-overall & P-non-linear \\
\hline MMSE & 0.221 & 0.111 & & $0.036^{*}$ & $0.020^{*}$ \\
AVLT-IR & 0.172 & 0.092 & & 0.144 & 0.107 \\
AVLT-SR & 0.204 & 0.313 & & 0.587 & 0.966 \\
AVLT-LR & 0.482 & 0.295 & & 0.894 & 0.767 \\
SDMT & 0.896 & 0.801 & & 0.661 & 0.483 \\
LMT & 0.988 & 0.936 & & 0.529 & 0.417 \\
TMTA & 0.633 & 0.435 & & 0.713 & 0.563 \\
TMTB & 0.559 & 0.356 & & 0.658 & 0.516 \\
DSTF & 0.957 & 0.990 & & 0.096 & 0.067 \\
DSTB & 0.650 & 0.771 & & 0.718 & 0.654 \\
PMT & 0.308 & 0.868 & & 0.161 & 0.712 \\
SCWT-IT & 0.924 & 0.866 & & 0.550 & 0.349 \\
\hline
\end{tabular}

Adjusted for age, sex, education, BMI, smoking, drinking, hypertension, hyperlipidemia, diabetes, CVD, and other nutrient patterns using restricted cubic spline (RCS) models. MMSE, Mini-Mental State Examination; AVLT-IR, Auditory Verbal Learning Testimmediate recall; AVLT-SR, Auditory Verbal Learning Test-short recall; AVLT-LR Auditory Verbal Learning Test-long recall; SDMT, Symbol Digit Modalities Test; LMT, Logical Memory Test; TMTA (B), Trail Making Test A (B); DSTF, digit span test forward; DSTB, digit span test backward; PMT, Picture Memories Test; SCWT-IT, Stroop Color-Word Test Interference Trial; BMI, body mass index; CVD, cerebrovascular diseases; Cl, confidence interval.

${ }^{\star} P<0.05,{ }^{\star \star} P<0.001$.

\section{Mediation Effect Between Cholesterol or Vitamin $B_{12}$ and Cognitive Function}

A total of 104 participants were selected in the subgroup to elucidate how cholesterol and vitamin $\mathrm{B}_{12}$ affect cognitive function. The characteristics between overall samples used in previous analysis $(n=2546)$ and subgroup $(n=104)$ were demonstrated in Supplementary Table 2. It was showed that there was a significant difference in sex, BMI or drink habit between subgroup and overall, so these variants were adjusted in the analysis subsequently.

In mediation analysis, the average causal mediation effect (ACME) between cholesterol or vitamin $\mathrm{B}_{12}$ and MMSE was not significant when 27-OHC, 24S-OHC, t-tau, or p-tau was used as a mediator $(P>0.05)$. None of the mediators had significance in the relationship between nutrients and cognition. The details are shown in Supplementary Table 3.

\section{DISCUSSIONS}

In this prospective study based on middle-aged and older adults, we examined the effect of nutrient patterns on cognitive function. Four nutrient patterns were identified, and only the CholesterolVitamin $\mathrm{B}_{12}$ pattern was associated with $\mathrm{MCI}(\mathrm{RR}=0.891$, $95 \% \mathrm{CI}=0.794,0.999)$. In multi-domain cognitive function, cholesterol and vitamin $B_{12}$ were positively correlated with PMT $(P=0.034, P=0.02)$. Furthermore, vitamin $B_{12}$ was non-linearly associated with MMSE and the dose-response curve emerged Ushaped $(P=0.02)$. Generally, this study provided a perspective 


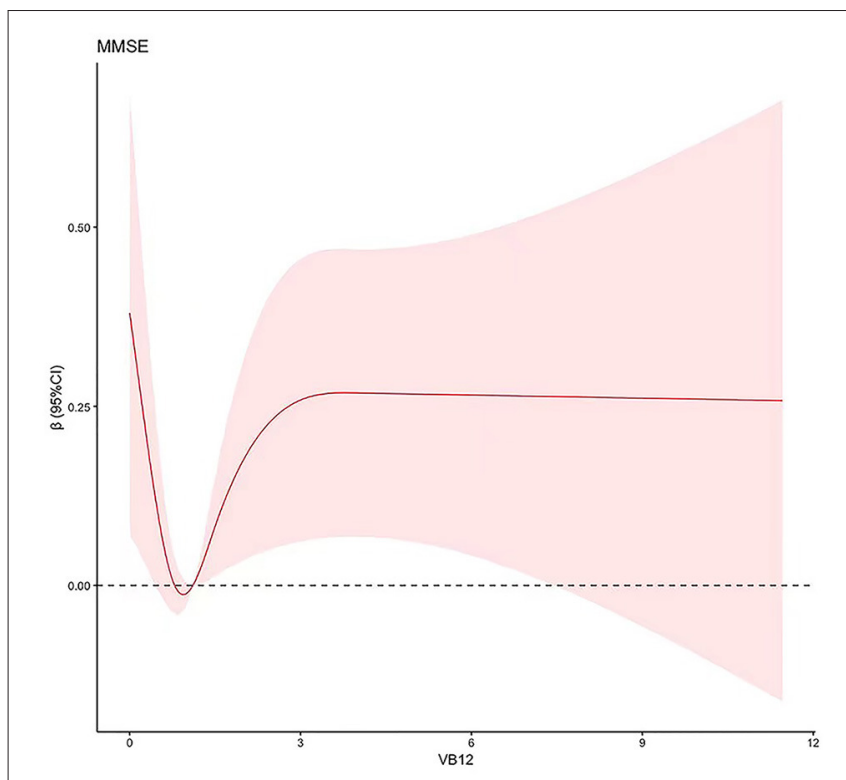

FIGURE 2 | Dose-response relationship between vitamin $\mathrm{B}_{12}$ and MMSE. Adjusted for age, sex, education, BMI, smoking, drinking, hypertension, hyperlipidemia, diabetes, CVD, and other nutrient patterns using RCS models. The shaded area represents the estimated relative risk and the $95 \% \mathrm{Cl}$. MMSE, Mini-Mental State Examination; BMI, body mass index; CVD, cerebrovascular diseases; $\mathrm{Cl}$, confidence interval.

that nutrition, as a modifiable factor, could be an applicable and critical strategy to improve cognitive function.

In this study, a diet rich in cholesterol was associated with better cognitive function. Similarly, a study based on Framingham Heart Study showed that lower total cholesterol was correlated with poorer performance on cognitive function, and another cohort study showed that cholesterol was associated with deceased risk of dementia (Elias et al., 2005; Mielke et al., 2005). Moreover, cholesterol intake restriction had been removed from the 2015-2020 Dietary Guidelines in America, which indicates that the effect of cholesterol on cognitive function is complicated, and it was still difficult to make a conclusion whether it was harmful or beneficial (Williams et al., 2015). In multi-domain cognitive function, dietary cholesterol was found to be associated with PMT in this study. PMT was a subtest of WMS-RC, which was used to assess visual memory function. Little research has examined the relationship between dietary cholesterol and visual memory function, and a study has found that a mild increase in low-density lipoprotein cholesterol or high-density lipoprotein cholesterol level is related to a better visual memory (Kinno et al., 2019; Guo et al., 2020). Therefore, maintaining moderate cholesterol consumption may be advisable for cognitive function.

In this study, vitamin $\mathrm{B}_{12}$ was non-linearly associated with MMSE when the intake dose was within a certain range, which suggested that it was important to ensure adequate consumption of vitamin $\mathrm{B}_{12}$. A cross-sectional study in Ireland also proposed that dietary vitamin $B_{12}$ was related with MMSE in older adults, indicating that vitamin $B_{12}$ may be a protective factor for cognitive function (O'Connor et al., 2020). Meantime, cross-sectional data from a nationally representative study in the United States showed that low vitamin $B_{12}$ was related to cognitive impairment independently among older adults (Molloy, 2020). Therefore, ensuring adequate vitamin $B_{12}$ intake might be an effective intervention to maintain cognitive function. In addition, in this study, vitamin $\mathrm{B}_{12}$ was related to a better performance in PMT. Although no direct evidence between vitamin $\mathrm{B}_{12}$ and visual memory had been found, a community-based cross-sectional study demonstrated that elevated homocysteine was associated with poorer performance in visual memory test (Ai-Vyrn et al., 2008). More studies are needed to be conducted on the possible mechanisms between vitamin $\mathrm{B}_{12}$ and visual memory function.

In this study, the cholesterol-vitamin $\mathrm{B}_{12}$ nutrient pattern was associated with MCI, which led us to consider the common mechanism of cholesterol and vitamin $\mathrm{B}_{12}$ in cognitive function. However, no statistical evidence for the hypothesis that cholesterol and vitamin $B_{12}$ could work together to affect cognitive function through cholesterol oxygenated derivatives or tau phosphorylation. First, mediation analysis can only identify mediators that had a linear mediation effect, but the biological process was complex, and the relationship might be nonlinear. Next, cholesterol and vitamin $\mathrm{B}_{12}$ may affect cognitive function through other mechanisms. For instance, it has been revealed that both cholesterol and vitamin $\mathrm{B}_{12}$ could influence cognitive function via $\mathrm{A} \beta$ metabolism (Alam et al., 2017; Wang et al., 2019). What is more, an inflammatory effect played an important role in the process of dietary cholesterol or vitamin $\mathrm{B}_{12}$ affecting cognitive function (Chen et al., 2018; Ma et al., 2019). Additionally, increasing research studies examined the role of epigenetics in biological mechanism of $\mathrm{AD}$. As an essential cofactor of homocysteine metabolism, vitamin $\mathrm{B}_{12}$ is integral to DNA methylation and consequently involved in the pathology of AD (Athanasopoulos et al., 2016). Recently, a cohort study in Scotland also found that cholesterol was associated with apolipoprotein E (APOE) methylation (Mur et al., 2020). Therefore, future studies may focus on exploring the common mechanism of cholesterol and vitamin $\mathrm{B}_{12}$ affecting cognitive function, especially in the role of epigenetics.

In this study, the nutrient pattern characterized as cholesterol and vitamin $\mathrm{B}_{12}$ was related to lower risk of MCI, and the possible biological explanation for this beneficial effect may come from the high loadings of cholesterol and vitamin $\mathrm{B}_{12}$. Mostly, dietary cholesterol and vitamin $\mathrm{B}_{12}$ are derived from meat, eggs, and milk. In this study, we explore the relationship of meat intake with cognitive function further, and it was found that meat intake was correlated with lower risk of MCI (Supplementary Table 4) and better performance of global cognitive function (Supplementary Table 5). Similarly, a longitudinal study on community dwellers in the state of New York also demonstrated that higher meat consumption was associated with better cognitive performance (Crichton et al., 2015). Meantime, a prospective study on oldest old Chinese population suggested that participants who consumed more meat were less likely to develop cognitive impairment (An et al., 2019). However, a cohort study from Sweden showed that adherence to a diet low in meat might contribute to healthy cognitive aging, the 
inconsistent results indicated that native conditions are needed to be considered in dietary instructions (Titova et al., 2013). The inconsistent evidence provides us a hypothesis that the effect of meat on cognitive function was complicated, and that it may be different regarding local conditions. In general, meat should be consumed appropriately, for it is a risk factor in other diseases such as cardiovascular disease.

This study has several strengths. First, the data in this study were based on a prospective study with a relatively large sample size. Second, cognitive function was assessed through multiple scales, such as verbal memory cognitive, attention/processing speed/executive function, flexibility cognitive function, and visual memory function, which would reflect cognitive function more comprehensively. Third, not only the linear effects of nutrients but also the non-linear effects on multi-domain cognitive function were evaluated. The limitations of this study are as follows: First, the number of APOE- $\varepsilon 4$ alleles is a major non-modifiable risk factor for $\mathrm{AD}$, but we did not measure the APOE genotype in this study. Recently, a national wide cross-sectional study in China observed that lifestyle such as dietary pattern was associated with cognitive function regardless of APOE genotype (Jin et al., 2021). In order to confirm the relationship between dietary intake and cognitive function, the research team will further explore it. Second, cognitive function was assessed by scales instead of clinical examination, and education level can significantly affect the performance in completing the scales. Thus, we adjusted education level in each statistical model to compensate for this limitation. Besides, the mediation effect of oxysterols or tau protein between cholesterol or vitamin $\mathrm{B}_{12}$ and MMSE was only examined by statistical analysis, but the biological process was complex, and the relationship might be non-linear. Therefore, animal experiments and in vitro studies are still needed to elucidate the molecular pathway and physiological regulation underlying the observed associations in the next step.

\section{CONCLUSIONS}

In summary, the study provided evidence that a nutrient pattern rich in cholesterol and vitamin $\mathrm{B}_{12}$ was associated with lower risk of MCI and better performance in visual memory function. Vitamin $\mathrm{B}_{12}$ was non-linearly associated with MMSE, and the curve emerged $U$-shaped when the intake was within a certain range, which suggested that it was essential to establish a doseresponse relationship for optimal dietary recommendations. Thus, ensuring appropriate cholesterol and vitamin $\mathrm{B}_{12}$ intake in the diet may be an effective strategy to prevent MCI in

\section{REFERENCES}

Ai-Vyrn, C., Robinson, D. J., Henry, O. C., Fiona, H., Irene, B., Robert, C., et al. (2008). Vascular biomarkers of cognitive performance in a community-based elderly population: the Dublin Healthy Ageing study. Age Ageing 37, 559-564. doi: 10.1093/ageing/afn144

Alam, P., Siddiqi, M. K., Chaturvedi, S. K., Zaman, M., and Khan, R. H. (2017). Vitamin B12 offers neuronal cell protection by inhibiting middle-aged and older adults. As a modifiable factor in cognitive function intervention, scientific dietary guidelines are critical.

\section{DATA AVAILABILITY STATEMENT}

The datasets analyzed during the current study are available from the corresponding author on reasonable request.

\section{ETHICS STATEMENT}

The studies involving human participants were reviewed and approved by the Ethics Committee of Capital Medical University (2013SY35). The patients/participants provided their written informed consent to participate in this study. Written informed consent was obtained from the individual(s) for the publication of any potentially identifiable images or data included in this article.

\section{AUTHOR CONTRIBUTIONS}

RX conceived and designed the study. LW and KL performed statistical analysis and wrote the manuscript. XZ, YW, WL, TW, $\mathrm{LH}$, and $\mathrm{MJ}$ helped in the collection of data and conducted the experiments. All authors contributed to the article and approved the submitted version.

\section{FUNDING}

This study was supported by the State Key Program of the National Natural Science Foundation of China (Grant No: 81330065 ) and the National Natural Science Foundation of China (Grant No: 81973021).

\section{ACKNOWLEDGMENTS}

The authors are indebted to the medical and ancillary staff of the Health Examination Center in Jincheng and Linyi and Beijing Chaoyang District Center for Disease Control and Prevention for the assistant in field survey. The authors would also like to thank all the participants for their time.

\section{SUPPLEMENTARY MATERIAL}

The Supplementary Material for this article can be found online at: https://www.frontiersin.org/articles/10.3389/fnagi. 2021.707958/full\#supplementary-material 
Bandaru, V. V., and Haughey, N. J. (2014). Quantitative detection of free 24Shydroxycholesterol, and 27-hydroxycholesterol from human serum. BMC Neurosci. 15:137. doi: 10.1186/s12868-014-0137-z

Bhat, N. R., and Thirumangalakudi, L. (2013). Increased tau phosphorylation and impaired brain insulin/IGF signaling in mice fed a high fat/high cholesterol diet. J. Alzheimers Dis. 36, 781-789. doi: 10.3233/JAD-2012-121030

Bondi, M. W., Serody, A. B., Chan, A. S., Eberson-Shumate, S. C., Delis, D. C., Hansen, L. A., et al. (2002). Cognitive and neuropathologic correlates of Stroop color-word test performance in Alzheimer's disease. Neuropsychology 16, 335-343. doi: 10.1037/0894-4105.16.3.335

Chen, Y., Yin, M., Cao, X., Hu, G., and Xiao, M. (2018). Pro- and antiinflammatory effects of high cholesterol diet on aged brain. Aging Dis. 9, 374-390. doi: 10.14336/AD.2017.0706

Chianese, R., Coccurello, R., Viggiano, A., Scafuro, M., and Meccariello, R. (2017). Impact of dietary fats on brain functions. Curr. Neuropharmacol. 16, 1059-1085. doi: 10.2174/1570159X15666171017102547

Clare, W., Jolene, H., Zoey, T., Salome, R., Lu, Y., Choon, O., et al. (2017). Relative validity and reproducibility of a food frequency questionnaire for assessing dietary intakes in a multi-ethnic Asian population using 24-h dietary recalls and biomarkers. Nutrients 9:1059. doi: 10.3390/nu9101059

Crichton, G. E., Elias, M. F., Davey, A., Alkerwi, A., and Dore, G. A. (2015). Higher cognitive performance is prospectively associated with healthy dietary choices: the Maine Syracuse Longitudinal Study. J. Prev. Alzheimers Dis. 2, 24-32. doi: $10.14283 /$ jpad.2015.39

Cui, L., Hou, N. N., Wu, H. M., Zuo, X., Lian, Y. Z., Zhang, C. N., et al. (2020). Prevalence of Alzheimer's disease and Parkinson's disease in China: an updated systematical analysis. Front. Aging Neurosci. 12:603854. doi: $10.3389 /$ fnagi.2020.603854

Darby, R. R., Brickhouse, M., Wolk, D. A., and Dickerson, B. C. (2017). Effects of cognitive reserve depend on executive and semantic demands of the task. J. Neurol. Neurosurg. Psychiatry 2017:jnnp-2017-315719. doi: 10.1136/jnnp-2017-315719

Desquilbet, L., and Mariotti, F. (2010). Dose-response analyses using restricted cubic spline functions in public health research. Stat. Med. 29, 1037-1057. doi: $10.1002 / \operatorname{sim} .3841$

Elias, P. K., Elias, M. F., D’Agostino, R. B., Sullivan, L. M., and Wolf, P. A. (2005). Serum cholesterol and cognitive performance in the Framingham Heart Study. Psychosom. Med. 67, 24-30. doi: 10.1097/01.psy.0000151745.67285.c2

Fiorini, R., Luzzi, S., and Vignini, A. (2020). Perspectives on mild cognitive impairment as a precursor of Alzheimer's disease. Neural Regen. Res. 15, 2039-2040. doi: 10.4103/1673-5374.282256

Guo, Y., Li, P., Ma, X., Huang, X., Liu, Z., Ren, X., et al. (2020). Association of circulating cholesterol level with cognitive function and mild cognitive impairment in the elderly: a community-based population study. Curr. Alzheimer Res. 17, 556-565. doi: 10.2174/1567205017666200810165758

Hong, Z., Ding, D., Zhou, Y., Chen, M., Liang, X., Guo, Q., et al. (2015). Auditory verbal learning test is superior to rey-osterrieth complex figure memory for predicting mild cognitive impairment to Alzheimer's Disease. Current Alzheimer Res. 12, 520-526. doi: 10.2174/1567205012666150530202729

Jia, L., Du, Y., Chu, L., Zhang, Z., Li, F., Lyu, D., et al. (2020). Prevalence, risk factors, and management of dementia and mild cognitive impairment in adults aged 60 years or older in China: a cross-sectional study. Lancet Public Health 5, e661-e671. doi: 10.1016/S2468-2667(20)30185-7

Jiang, Y. W., Sheng, L. T., Pan, X. F., Feng, L., Yuan, J. M., Pan, A., et al. (2020). Midlife dietary intakes of monounsaturated acids, n- 6 polyunsaturated acids, and plant-based fat are inversely associated with risk of cognitive impairment in older Singapore Chinese adults. J. Nutr. 150, 901-909. doi: 10.1093/jn/ nxz325

Jin, X., He, W., Zhang, Y., Gong, E., Niu, Z., Ji, J., et al. (2021). Association of APOE $\varepsilon 4$ genotype and lifestyle with cognitive function among Chinese adults aged 80 years and older: A cross-sectional study. PLoS Med. 18:e1003597. doi: 10.1371/journal.pmed.1003597

Kinno, R., Mori, Y., Kubota, S., Nomoto, S., Futamura, A., Shiromaru, A., et al. (2019). High serum high-density lipoprotein-cholesterol is associated with memory function and gyrification of insular and frontal opercular cortex in an elderly memory-clinic population. Neuroimage Clin. 22:101746. doi: 10.1016/j.nicl.2019.101746
Krakowski, M., and Czobor, P. (2011). Cholesterol and cognition in schizophrenia: a double-blind study of patients randomized to clozapine, olanzapine and haloperidol. Schizophr. Res. 130, 27-33. doi: 10.1016/j.schres.2011.04.005

Lo, K., Liu, Q., Madsen, T., Rapp, S., Chen, J. C., Neuhouser, M., et al. (2019). Relations of magnesium intake to cognitive impairment and dementia among participants in the Women's Health Initiative Memory Study: a prospective cohort study. BMJ Open 9:e030052. doi: 10.1136/bmjopen-2019-030052

Ma, F., Zhou, X., Li, Q., Zhao, J., Song, A., An, P., et al. (2019). Effects of folic acid and vitamin B12, alone and in combination on cognitive function and inflammatory factors in the elderly with mild cognitive impairment: a single-blind experimental design. Curr. Alzheimer Res. 16, 622-632. doi: $10.2174 / 1567205016666190725144629$

Makura-Kankwende, C. B. T., Gradidge, P. J., Crowther, N. J., Norris, S. A., and Chikowore, T. (2020). Nutrient patterns and body composition parameters of black South African women. Nutrients 13:6. doi: 10.3390/nu13010006

Mielke, M. M., Zandi, P. P., Sjögren, M., Gustafson, D., Ostling, S., Steen, B., et al. (2005). High total cholesterol levels in late life associated with a reduced risk of dementia. Neurology 64, 1689-1695. doi: 10.1212/01.WNL.0000161870.78572.A5

Molloy, A. M. (2020). Adverse effects on cognition caused by combined low vitamin B-12 and high folate status-we must do better than a definite maybe! Am. J. Clin. Nutr. 112, 1422-1423. doi: 10.1093/ajen/nqaa286

Mur, J., McCartney, D. L., Walker, R. M., Campbell, A., Bermingham, M. L., Morris, S. W., et al. (2020). DNA methylation in APOE: the relationship with Alzheimer's and with cardiovascular health. Alzheimers Dement. 6:e12026. doi: $10.1002 / \operatorname{trc} 2.12026$

O'Connor, D. M. A., Laird, E. J., Carey, D., O’Halloran, A. M., Clarke, R., Kenny, R. A., et al. (2020). Plasma concentrations of vitamin B(12) and folate and global cognitive function in an older population: cross-sectional findings from The Irish Longitudinal Study on Ageing (TILDA). Br. J. Nutr. 124, 602-610. doi: $10.1017 /$ S0007114520001427

Palta, P., Chen, H., Deal, J. A., Sharrett, A. R., Gross, A., Knopman, D., et al. (2018). Olfactory function and neurocognitive outcomes in old age: the Atherosclerosis Risk in Communities Neurocognitive Study. Alzheimers Dement. 14, 1015-1021. doi: 10.1016/j.jalz.2018.02.019

Physical Status (1995). The use and interpretation of anthropometry. Report of a WHO Expert Committee. World Health Organ. Tech. Rep. Ser. (1995) $854,1-452$.

Poulose, S. M., Miller, M. G., Tammy, S., Barbara, S. H. Nutritional factors affecting adult neurogenesis and cognitive function. Adv. Nutr. (2017) 8, 804-811. doi: 10.3945/an.117.016261

Prinelli, F., Fratiglioni, L., Kalpouzos, G., Musicco, M., Adorni, F., Johansson, I., et al. (2019). Specific nutrient patterns are associated with higher structural brain integrity in dementia-free older adults. Neuroimage 199, 281-288. doi: 10.1016/j.neuroimage.2019.05.066

Rong, H., Xi, Y., An, Y., Tao, L., Zhang, X., Yu, H., et al. (2017). The correlation between early stages of life exposed to chinese famine and cognitive decline in adulthood: nutrition of adulthood plays an important role in the link? Front. Aging Neurosci. 9:444. doi: 10.3389/fnagi.2017.00444

Scarmeas, N., Anastasiou, C. A., and Yannakoulia, M. (2018). Nutrition and prevention of cognitive impairment. Lancet Neurol. 17, 1006-1015. doi: $10.1016 /$ S1474-4422(18)30338-7

Shi, Z., Li, M., Wang, Y., Liu, J., and El-Obeid, T. (2019). High iron intake is associated with poor cognition among Chinese old adults and varied by weight status-a 15-y longitudinal study in 4852 adults. Am. J. Clin. Nutr. 109, 109-116. doi: 10.1093/ajen/nqy254

Smith, A. D., and Refsum, H. (2016). Homocysteine, B vitamins, and cognitive impairment. Ann. Rev. Nutr. 36, 211-239. doi: 10.1146/annurev-nutr-071715-050947

Tang, Y., Liu, H. L., Min, L. X., Yuan, H. S., Guo, L., Han, P. B., et al. (2019). Serum and cerebrospinal fluid tau protein level as biomarkers for evaluating acute spinal cord injury severity and motor function outcome. Neural Regen. Res. 14, 896-902. doi: 10.4103/1673-5374.249238

Titova, O. E., Ax, E., Brooks, S. J., Sjögren, P., Cederholm, T., Kilander, L., et al. (2013). Mediterranean diet habits in older individuals: associations with cognitive functioning and brain volumes. Exp. Gerontol. 48, 1443-1448. doi: 10.1016/j.exger.2013.10.002 
Vauzour, D., Robles, M. C., Miquel-Kergoat, S., Andres-Lacueva, C., Bánáti, D., Barberger-Gateau, P., et al. (2017). Nutrition for the ageing brain: towards evidence for an optimal diet. Ageing Res. Rev. 35, 222-240. doi: 10.1016/j.arr.2016.09.010

Wang, C., An, Y., Yu, H., Feng, L., Liu, Q., Lu, Y., et al. (2016b). Association between exposure to the chinese famine in different stages of early life and decline in cognitive functioning in adulthood. Front. Behav. Neurosci. 10:146. doi: $10.3389 /$ fnbeh. 2016.00146

Wang, C., Shou, Y., Pan, J., Du, Y., Liu, C., and Wang, H. (2019). The relationship between cholesterol level and Alzheimer's diseaseassociated APP proteolysis/A $\beta$ metabolism. Nutr. Neurosci. 22, 453-463. doi: 10.1080/1028415X.2017.1416942

Wang, H. L., Wang, Y. Y., Liu, X. G., Kuo, S. H., Liu, N., Song, Q. Y., et al. (2016a). Cholesterol, 24-hydroxycholesterol, and 27-hydroxycholesterol as surrogate biomarkers in cerebrospinal fluid in mild cognitive impairment and Alzheimer's disease: a meta-analysis. J. Alzheimers Dis. 5, 45-55. doi: 10.3233/JAD-150734

Wei, M., Shi, J., Li, T., Ni, J., Zhang, X., Li, Y., et al. (2018). Diagnostic accuracy of the Chinese version of the trail-making test for screening cognitive impairment. J. Am. Geriatr. Soc. 66, 92-99. doi: 10.1111/jgs. 15135

Williams, K. A. Sr., Krause, A. J., Shearer, S., and Devries, S. (2015). The 2015 dietary guidelines advisory committee report concerning dietary cholesterol. Am. J. Cardiol. 116, 1479-1480. doi: 10.1016/j.amjcard.2015. 07.077

Wong, J. C., Scott, T., Wilde, P., Li, Y. G., Tucker, K. L., and Gao, X. (2016). Food insecurity is associated with subsequent cognitive decline in the Boston Puerto Rican health study. J. Nutr. 146, 1740-1745. doi: 10.3945/jn.115.2 28700

Xiong, X., Zhang, L., Hao, Y., Zhang, P., and Liu, G. (2019). Urban dietary changes and linked carbon footprint in China: a case study of Beijing. J. Environ. Manag. 255:109877. doi: 10.1016/j.jenvman.2019.109877

Xue, J., Li, J., Liang, J., and Chen, S. (2018). The prevalence of mild cognitive impairment in China: a systematic review. Aging Dis. 9, 706-715. doi: 10.14336/AD.2017.0928
Yin, Z., Chen, J., Zhang, J., Ren, Z., Dong, K., Kraus, V. B., et al. (2018) Dietary patterns associated with cognitive function among the older people in underdeveloped regions: finding from the NCDFaC study. Nutrients 10 . doi: 10.3390/nu10040464

Yu, A., Lingli, F., Xiaona, Z., Ying, W., Yushan, W., Lingwei, T., et al. (2018). Patterns of cognitive function in middle-aged and elderly Chinese adults-findings from the EMCOA study. Alzheimers Res. Ther. 10:93. doi: 10.1186/s13195-018-0421-8

Zhang, Y., Chen, J., Qiu, J., Li, Y., Wang, J., and Jiao, J. (2016). Intakes of fish and polyunsaturated fatty acids and mild-to-severe cognitive impairment risks: a dose-response meta-analysis of 21 cohort studies. Am. J. Clin. Nutr. 103, 330-340. doi: 10.3945/ajcn.115.124081

Zhou, M., Wang, H., Zeng, X., Yin, P., Zhu, J., Chen, W., et al. (2019). Mortality, morbidity, and risk factors in China and its provinces, 1990-2017: a systematic analysis for the Global Burden of Disease Study 2017. Lancet 394, 1145-1158. doi: 10.1016/S0140-6736(19)30427-1

Conflict of Interest: The authors declare that the research was conducted in the absence of any commercial or financial relationships that could be construed as a potential conflict of interest.

Publisher's Note: All claims expressed in this article are solely those of the authors and do not necessarily represent those of their affiliated organizations, or those of the publisher, the editors and the reviewers. Any product that may be evaluated in this article, or claim that may be made by its manufacturer, is not guaranteed or endorsed by the publisher.

Copyright (c) 2021 Wang, Liu, Zhang, Wang, Liu, Wang, Hao, Ju and Xiao. This is an open-access article distributed under the terms of the Creative Commons Attribution License (CC BY). The use, distribution or reproduction in other forums is permitted, provided the original author(s) and the copyright owner(s) are credited and that the original publication in this journal is cited, in accordance with accepted academic practice. No use, distribution or reproduction is permitted which does not comply with these terms. 